Bull. Austral. Math. Soc.

VoL. 43 (1991) [387-392]

\title{
MONOCHROMATIC SOLUTIONS TO EQUATIONS WITH UNIT FRACTIONS
}

\author{
TOM C. BROWN AND VOIJTECh RÖDL
}

Our main result is that if $G\left(x_{1}, \cdots, x_{n}\right)=0$ is a system of homogeneous equations such that for every partition of the positive integers into finitely many classes there are distinct $y_{1}, \cdots, y_{n}$ in one class such that $G\left(y_{1}, \cdots, y_{n}\right)=0$, then, for every partition of the positive integers into finitely many classes there are distinct $z_{1}, \cdots, z_{n}$ in one class such that

$$
G\left(\frac{1}{z_{1}}, \cdots, \frac{1}{z_{n}}\right)=0
$$

In particular, we show that if the positive integers are split into $r$ classes, then for every $n \geqslant 2$ there are distinct positive integers $x_{1}, x_{1}, \ldots, x_{n}$ in one class such that

$$
\frac{1}{x_{0}}=\frac{1}{x_{1}}+\cdots+\frac{1}{x_{n}}
$$

We also show that if $\left[1, n^{0}-\left(n^{2}-n\right)^{2}\right]$ is partitioned into two classes, then some class contains $x_{0}, x_{1}, \ldots, x_{n}$ such that

$$
\frac{1}{x_{0}}=\frac{1}{x_{1}}+\ldots+\frac{1}{x_{n}} \text {. }
$$

(Here, $x_{0}, x_{2}, \ldots, x_{n}$ are not necessarily distinct.)

\section{INTRODUCTION}

In their monograph [1], Erdös and Graham list a large number of questions concerned with equations with unit fractions. In fact, a whole chapter is devoted to this topic. One of their questions, still open, is the following.

In the positive integers, let

$$
H_{m}=\left\{\left\{x_{1}, \cdots, x_{m}\right\}: \sum_{k=1}^{m} 1 / x_{k}=1,0<x_{1}<\cdots<x_{m}\right\},
$$

Received 29 May 1990

The first author was partially supported by NSERC.

Copyright Clearance Centre, Inc. Serial-fee code: 0004-9729/91 SA2.00+0.00. 
and let $H$ denote the union of all the $H_{m}, m \geqslant 1$. Now arbitrarily split the positive integers into $r$ classes. It is true that some element of $H$ is contained entirely in one class?

In this note we show (Corollary 2.4 below) that if one does not require all the $x_{k}$ 's to be distinct, but only many of the $x_{k}$ 's to be distinct, then the answer to the corresponding question is yes. More precisely, we show that if the positive integers are split into $r$ classes, then for every $n$ there exist $m \geqslant n$ and $x_{1}, \cdots, x_{m}$ (not necessarily distinct) in one class such that $\left|\left\{x_{1}, \cdots, x_{m}\right\}\right| \geqslant n$ and $\sum_{k=1}^{m} 1 / x_{k}=1$.

We actually show (Corollary 2.3 below) something stronger, namely that if the positive integers are split into $r$ classes, then for every $n \geqslant 2$ there are distinct positive integers $x_{0}, x_{1}, \ldots, x_{n}$ in one class such that

$$
\frac{1}{x_{0}}=\frac{1}{x_{1}}+\cdots \frac{1}{x_{n}}
$$

(The preceding result then follows by taking $x_{0}$ copies of each of $x_{1}, \cdots, x_{n}$ )

Our main result (Theorem 2.1) is that if $G\left(x_{1}, \cdots, x_{n}\right)=0$ is a system of homogeneous equations such that for every partition of the positive integers into finitely many classes there are distinct $y_{1}, \cdots, y_{n}$ in one class such that $G\left(y_{1}, \cdots, y_{n}\right)=0$, then, for every partition of the positive integers into finitely many classes there are distinct $z_{1}, \cdots, z_{n}$ in one class such that

$$
G\left(\frac{1}{z_{1}}, \cdots, \frac{1}{z_{n}}\right)=0 .
$$

We also prove (Theorem 2.5) the following quantitative result. Let $f(n)$ be the smallest $N$ such that if $[1, N]$ is partitioned into two classes, then some class contains $x_{0}, x_{1}, \ldots, x_{n}$ such that $1 / x_{0}=1 / x_{1}+\cdots+1 / x_{n}$. (Here, $x_{0}, x_{1}, \ldots, x_{n}$ are not necessarily distinct.) Then

$$
f(n) \leqslant n^{6}-\left(n^{2}-n\right)^{2} \text {. }
$$

\section{RESULTS}

From now on we shall use the terminology of colourings rather than partitions. That is, instead of "partition into $r$ classes" we say " $r$-colouring," and instead of "there are distinct $y_{1}, \cdots, y_{n}$ in one class such that $G\left(y_{1}, \cdots, y_{n}\right)=0$ " we say "there is a monochromatic solution of $G\left(y_{1}, \cdots, y_{n}\right)=0$ in distinct $y_{1}, \cdots, y_{n}$ ".

THEOREM 2.1. Let $G\left(x_{1}, \cdots, x_{n}\right)=0$ be a system of homogeneous equations such that for every finite colouring of the positive integers there is a monochromatic solution of $G\left(y_{1}, \cdots, y_{n}\right)=0$ in distinct $y_{1}, \cdots, y_{n}$. Then, for every finite colouring 
of the positive integers there is a monochromatic solution of $G\left(1 / z_{1}, \cdots, 1 / z_{n}\right)=0$ in distinct $z_{1}, \cdots, z_{n}$

Proof: Let $r$ be given, and consider a system $G\left(x_{1}, \cdots, x_{n}\right)=0$ of homogeneous equations such that for every $r$-colouring of the positive integers there is a monochromatic solution of $G\left(y_{1}, \cdots, y_{n}\right)=0$ in distinct $y_{1}, \cdots, y_{n}$. By a standard compactness argument, there exists a positive integer $T$ such that if $[1, T]$ is $r$-roloured, there is a monochromatic solution to $G\left(y_{1}, \cdots, y_{n}\right)=0$ in distinct $y_{1}, \cdots, y_{n}$.

Let $S$ denote the least common multiple of $1,2, \cdots, T$. We show that for every $r$-colouring of $[1, S]$ there is a monochromatic solution of $G\left(1 / z_{1}, \cdots, 1 / z_{n}\right)=0$ in distinct $z_{1}, \cdots, z_{n}$.

To do this, let

$$
c:[1, S] \rightarrow[1, r]
$$

be an arbitrary $r$-colouring of $[1, S]$.

Define an $r$-colouring $\bar{c}$ of $[1, T]$ by setting

$$
\bar{c}(x)=c(S / x), 1 \leqslant x \leqslant T .
$$

By the definition of $T$, there is a solution of $G\left(y_{1}, \cdots, y_{n}\right)=0$ in distinct $y_{1}, \cdots, y_{n}$ such that

$$
\bar{c}\left(y_{1}\right)=\bar{c}\left(y_{2}\right)=\cdots=\bar{c}\left(y_{n}\right) .
$$

By the definition of $\bar{c}$, this means that

$$
c\left(S / y_{1}\right)=c\left(S / y_{2}\right)=\cdots=c\left(S / y_{n}\right) .
$$

Setting $z_{i}=S / y_{i}, 1 \leqslant i \leqslant n$, we have that $z_{1}, \cdots, z_{n}$ are distinct, are monochromatic relative to the colouring $c$ of $[1, S]$, and that

$$
G\left(\frac{1}{z_{1}}, \cdots, \frac{1}{z_{n}}\right)=0 .
$$

Omitting all references to distinctness, one gets the following.

THEOREM 2.1A. Let $G\left(x_{1}, \cdots, x_{n}\right)=0$ be a system of homogeneous equations such that for every finite colouring of the positive integers there is a monochromatic solution of $G\left(x_{1}, \cdots, x_{n}\right)=0$. Then, for every finite colouring of the positive integers there is a monochromatic solution of $G\left(1 / z_{1}, \cdots, 1 / z_{n}\right)=0$.

Corollary 2.2. Let $a_{1}, \cdots, a_{m}, b_{1}, \cdots, b_{n}$ be positive integers such that

(1) some non-empty subset of the $a_{i}$ 's has the same sum as some non-empty subset of the $b_{j}$ 's and

(2) there exist distinct integers $u_{1}, \cdots, u_{m}, v_{1}, \cdots, v_{n}$ such that $a_{1} u_{1}+$ $\cdots+a_{m} u_{m}=b_{1} v_{1}+\cdots+b_{n} v_{n}$. 
Then, given any r-colouring of the positive integers, there is a monochromatic solution of

$$
\frac{a_{1}}{x_{1}}+\cdots+\frac{a_{m}}{x_{m}}=\frac{b_{1}}{y_{1}}+\cdots+\frac{b_{n}}{y_{n}}
$$

in distinct $x_{1}, \cdots, x_{m}, y_{1}, \cdots, y_{n}$.

Proof: Let $a_{1}, \cdots, a_{m}, b_{1}, \cdots, b_{n}$ satisfy conditions (1) and (2). According to Rado's theorem [3] (also see [2, p.59]), the equation

$$
a_{1} x_{1}+\cdots+a_{m} x_{m}=b_{1} y_{1}+\cdots+b_{n} y_{n}
$$

will always have a monochromatic solution $x_{1}, \cdots, x_{m}, y_{1}, \cdots, y_{n}$, for every $r$ colouring of the positive integers, because of condition (1). The additional condition (2) is enough (see $\left[2\right.$, p.62 Corollary $\left.\left.8 \frac{1}{2}\right]\right)$ to ensure that the equation

$$
a_{1} x_{1}+\cdots+a_{m} x_{m}=b_{1} y_{1}+\cdots+b_{n} y_{n}
$$

will always have a monochromatic solution $x_{1}, \cdots, x_{m}, y_{1}, \cdots, y_{n}$, in distinct $x_{1}, \cdots, x_{m}, y_{1}, \cdots, y_{n}$. Theorem 2.1 now applies.

COROLLARY 2.3. Let an arbitrary $r$-colouring of the positive integers be given. Let $n, a$ be positive integers, with $n \geqslant 2$ and $1 \leqslant a \leqslant n$. Then the equation

$$
\frac{a}{x_{0}}=\frac{1}{x_{1}}+\cdots+\frac{1}{x_{n}}
$$

has a monochromatic solution in distinct $x_{0}, x_{1}, \cdots, x_{n}$.

Proof: This follows immediately from Corollary 2.2 .

COROLLARY 2.4. Let an arbitrary $r$-colouring of the positive integers be given. Then for every $n$ there exist $m \geqslant n$ and monochromatic $x_{1}, \cdots, x_{m}$ (not necessarily distinct) such that $\left|\left\{x_{1}, \cdots, x_{m}\right\}\right| \geqslant n$ and $\sum_{k=1}^{m} 1 / x_{k}=1$.

Proof: Apply Corollary 2.3 (with $a=1$ ) and take $x_{0}$ copies of each of $x_{1}, \cdots, x_{m}$.

THEOREM 2.5. For each $n \geqslant 2$, let $f(n)$ be the smallest $N$ such that if $[1, N]$ is partitioned into two classes, then some class contains $x_{0}, x_{1}, \cdots, x_{n}$ such that

$$
\frac{1}{x_{0}}=\frac{1}{x_{1}}+\cdots+\frac{1}{x_{n}} .
$$

(Here, $x_{0}, x_{1}, \cdots, x_{n}$ are not necessarily distinct.) Then

$$
f(n) \leqslant n^{6}-\left(n^{2}-n\right)^{2} .
$$


Proof: The proof is by contradiction. Fix $n \geqslant 2$, let $N=n^{6}-\left(n^{2}-n\right)^{2}$, and suppose throughout the proof that $c:[1, N] \rightarrow\{1,2\}$ is some fixed 2 -colouring of $[1, N]$ for which there does not exist any monochromatic solution of

$$
\frac{1}{x_{0}}=\frac{1}{x_{1}}+\cdots+\frac{1}{x_{n}}
$$

LEMMA 2.6. (a) If $n x \leqslant N$ then $c(n x) \neq c(x)$.

(b) If $n^{2} x \leqslant N$ then $c\left(n^{2} x\right)=c(x)$.

Proof: Part (a) follows from $1 / x=1 /(n x)+\cdots+1 /(n x)$. Part (b) follows from part (a).

LеммA 2.7. If $n^{2}\left(n^{2}+n-1\right) x \leqslant N$, then $c\left(\left(n^{2}+n-1\right) x\right) \neq c(x)$.

PROOF: This follows from

$$
\frac{1}{n^{2} x}=\frac{1}{\left(n^{2}+n-1\right) x}+(n-1) \frac{1}{n^{2}\left(n^{2}+n-1\right) x}
$$

and Lemma 2.6.

LEMMA 2.8. If $n^{2}\left(n^{2}-n+1\right) x \leqslant N$, then $c\left(\left(n^{2}-n+1\right) x\right) \neq c(x)$.

Proof: This follows from

$$
\frac{1}{\left(n^{2}-n+1\right) x}=\frac{1}{n^{2} x}+(n-1) \frac{1}{n^{2}\left(n^{2}-n+1\right) x}
$$

and Lemma 2.6.

LEMMA 2.9. If $n^{2}\left(n^{2}+n-1\right) x \leqslant N$, then $c((n+1) x)=c(x)$.

Proof: This follows from

$$
\frac{1}{n(n+1) x}=\frac{1}{\left(n^{2}+n-1\right)(n+1) x}+(n-1) \frac{1}{\left(n^{2}+n-1\right) n x},
$$

and Lemmas 2.6 and 2.7.

LEMMA 2.10. If $n^{2}\left(n^{2}+n-1\right)\left(n^{2}-n+1\right) x \leqslant N$, then $c(2 x)=c(x)$.

Proof: This follows from

$$
\frac{1}{\left(n^{2}-n+1\right) 2 x}=\frac{1}{\left(n^{2}+n-1\right) 2 x}+(n-1) \frac{1}{\left(n^{2}+n-1\right)\left(n^{2}-n+1\right) x}
$$

and Lemmas 2.7 and 2.8 .

Finally, Theorem 2.5 is proved by observing that

$$
\frac{1}{2 \cdot 1}=\frac{1}{(n+1) \cdot 1}+(n-1) \frac{1}{2(n+1) \cdot 1}
$$

and by Lemmas 2.9 and $2.10, c(2 \cdot 1)=c((n+1) \cdot 1)=c(2(n+1) \cdot 1)=c(1)$, a contradiction. 
REMARK. The authors have learned that Hanno Lefmann (Bielefeld) has independently obtained results which include our Theorem $2.1 \mathrm{a}$.

\section{REFERENCES}

[1] P. Erdös and R.L. Graham, Old and new problems and results in combinatorial number theory, L'enseignement mathematique (Universite de Geneve, Geneva, 1980).

[2] R.L. Graham, B.L. Rothschild and J.H. Spencer, Ramsey theory (John Wiley and Sons, New York, 1980).

[3] R. Rado, 'Studien zur Kombinatorik', Math. Z. 36 (1933), 424-480.

Department of Mathematics and Statistics

Simon Fraser University

Burnaby BC

Canada V5A 156
Department of Mathematics and Computer Science

Emory University

Atlanta GA 30322-9998

United States of America 